\title{
The Honeycomb Conjecture
}

\author{
T. C. Hales \\ Department of Mathematics, University of Michigan, \\ Ann Arbor, MI 48109, USA
}

\begin{abstract}
This article gives a proof of the classical honeycomb conjecture: any partition of the plane into regions of equal area has perimeter at least that of the regular hexagonal honeycomb tiling.
\end{abstract}

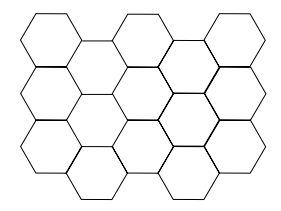

\section{Introduction}

Around 36 B.C., Marcus Terentius Varro, in his book on agriculture, wrote about the hexagonal form of the bee's honeycomb [V]. There were two competing theories of the hexagonal structure. One theory held that the hexagons better accommodated the bee's six feet. The other theory, supported by the mathematicians of the day, was that the structure was explained by an isoperimetric property of the hexagonal honeycomb. Varro wrote, "Does not the chamber in the comb have six angles ... The geometricians prove that this hexagon inscribed in a circular figure encloses the greatest amount of space."

The origin of this problem is somewhat obscure. Varro was aware of it long before Pappus of Alexandria, who mentions it in his fifth book [P1]. Much of Book V follows Zenodorus's much earlier work Isometric Figures (ca 180 B.C.). However, only fragments of Zenodorus's book remain, and it is not known whether the honeycomb is discussed there.

The argument in Pappus is incomplete. In fact it involves nothing more than a comparison of three suggestive cases. It was known to the Pythagoreans that only three regular 
polygons tile the plane: the triangle, the square, and the hexagon. Pappus states that if the same quantity of material is used for the constructions of these figures, it is the hexagon that will be able to hold more honey. Pappus's reason for restricting to the three regular polygons that tile are not mathematical (bees avoid dissimilar figures). He also excludes gaps between the cells of the honeycomb without mathematical argument. If the cells are not contiguous "foreign matter could enter the interstices between them and so defile the purity of their produce" [H, p. 390].

In part because of the isoperimetric property of the honeycomb, there is is a vast literature through the centuries mentioning the bee as a geometer. Thompson devotes nearly 20 pages to the literature on the bee's cell in [T2]. Some background also appears in [K1, Intro.], [W3] and [W1]. During the 18th century, the mathematical architecture of the honeycomb was viewed as evidence of a great teleological tendency of the universe. Darwin explained the same structures by natural selection. "That motive power of the process of natural selection having been economy of wax; that individual swarm that wasted least honey in the secretion of wax, having succeeded best" [D, p. 235].

The honeycomb problem has never been solved, except under special hypotheses. An unsolved special case of the problem is attributed to Steinhaus in Problem C15 of [CFG]. Extending Pappus's results, in 1943, L. Fejes Tóth proved the honeycomb conjecture under the hypothesis that the cells are convex [FT1]. L. Fejes Tóth predicted that a proof of the honeycomb conjecture without the convexity hypothesis would "involve considerable difficulties" [FT2, p. 183]. Elsewhere, he writes about his proof for convex cells, "There is no doubt that the same is true for general cells. Nevertheless, this conjecture resisted all attempts at proving it" [FT3]. This paper gives a proof without the assumption of convexity.

Convexity is a highly restrictive hypothesis. This hypothesis immediately forces the boundaries of the cells to be polygons. By the isoperimetric inequality, we expect potential counterexamples to be regions bounded by circular arcs. One of the two regions bounded by a positively curved arc will not be convex. Thus, the assumption of convexity eliminates at once almost all the candidates that should be studied the most closely.

The geometrical properties of the three-dimensional honeycomb cells have also been studied extensively. The three-dimensional honeycomb cell is a hexagonal prism built on a base of three congruent rhombuses. The shape of the rhombic base of the threedimensional cell suggested the rhombic dodecahedron to Kepler, the Voronoi cell of the face-centered cubic lattice. During the 18th century, many mathematicians studied the isoperimetric properties of the base of the cells. MacLaurin, in his analysis of the honeycomb, wrote, in 1743, "The sagacity of the bees in making their cells of an hexagonal form, has been admired of old." "The cells, by being hexagonal, are the most capacious, in proportion to their surface, of any regular figures that leave no interstices between them, and at the same time admit of the most perfect bases" [M1]. In a reversal of MacLaurin's conclusions and upsetting the prevailing opinion, L. Fejes Tóth discovered that the three-dimensional honeycomb cell is not the most economical (that is, it is not surface area minimizing) [FT3].

The honeycomb conjecture is the two-dimensional version of the three-dimensional Kelvin problem. The Kelvin problem asks for the surface minimizing partition of space into cells of equal volume. According to Lhuilier's memoir of 1781, the problem has been described as one of the most difficult in geometry [L1, p. 281]. The solution 
proposed by Kelvin is a natural generalization of the hexagonal honeycomb in two dimensions. Take the Voronoi cells of the dual lattice of the lattice giving the densest sphere packing. In two dimensions this is the honeycomb arrangement. In three dimensions this gives truncated octahedra, the Voronoi cells of the body-centered cubic. A small deformation of the faces produces a minimal surface, which is Kelvin's proposed solution.

Phelan and Weaire produced a remarkable counterexample to the Kelvin conjecture. As a result, the honeycomb problem in two dimensions has come under increased scrutiny, and the need for a solution has become more acute. Morgan remarks, "In 1994, D. Weaire and R. Phelan improved on Lord Kelvin's candidate for the least-area way to partition space into regions of unit volume. Contrary to popular belief, even the planar question remains open" [M4].

It seems that the honeycomb is minimal with respect to various optimization problems. Even the classical problem can be expressed as a minimization of perimeter for fixed areas or as a maximization of areas for fixed perimeters. The first of the two presents greater difficulties and is treated here. (See Section 26 of [FT2]).

Morgan states several versions and points out that the versions are not known to be equivalent. Here the situation is similar to the sphere packing problem, which also has several competing versions (find the densest, the solid, or tight packings [CS], the finitely stable or the uniformly stable sphere packings [BBC]). (Also see [K2].) These notions have isoperimetric analogues. However, here the situation is even more perplexing because there is no upper bound on the diameter of the cells of a partition of the plane into equal areas. In this paper we follow the first approach outlined by Morgan (Section 2 of his paper). A topic for future research might be to determine to what extent the methods of this paper can be adapted to the other optimization problems.

Steiner's proofs of the isoperimetric problem were criticized by Weierstrass because they did not prove the existence of a solution. Today, general theorems assuring the existence and regularity of solutions to isoperimetric problems are available. (See [T1], [A], [M2], and compare [B].) This paper depends on these results, assuring the existence of a solution to our isoperimetric problems.

To solve the problem, we replace the planar cluster with a cluster on a flat torus. The torus has the advantages of compactness and a vanishing Euler characteristic. This part of the proof is reminiscent of [FT1], which transports the planar cluster to a sphere. The key inequality, called the hexagonal isoperimetric inequality, appears in Theorem 4. It asserts that a certain functional is uniquely minimized by a regular hexagon of area 1. The isoperimetric properties of the functional force the minimizing figure to be convex. A penalty term prevents the solution from becoming too "round." The optimality of the hexagonal honeycomb results.

\section{Statement of the Theorem}

We follow [M4] in the formulation of Theorem 1-A. Let $\pi_{N}=N \tan (\pi / N)$ be the isoperimetric constant for a regular $N$-gon. That is, $4 \pi_{N}$ is the ratio of the circumference squared to the area of a regular $N$-gon. The particularly important case, the perimeter 
$2 \sqrt{\pi_{6}}=2 \sqrt[4]{12}$ of a regular hexagon with unit area, is used frequently. Let $B(0, r)$ be a disk of radius $r$ at the origin.

Theorem 1-A (Honeycomb Conjecture). Let $\Gamma$ be a locally finite graph in $\mathbb{R}^{2}$, consisting of smooth curves, and such that $\mathbb{R}^{2} \backslash \Gamma$ has infinitely many bounded connected components, all of unit area. Let $C$ be the union of these bounded components. Then

$$
\limsup _{r \mapsto \infty} \frac{\operatorname{perim}(C \cap B(0, r))}{\operatorname{area}(C \cap B(0, r))} \geq \sqrt[4]{12} .
$$

Equality is attained for the regular hexagonal tile.

The limit is insensitive to compact alterations. Therefore, there is no uniqueness statement for the theorem in this form. The uniqueness of the hexagonal tile appears in the compact version of Theorem 3 below.

Theorem 1-A has stronger hypotheses than necessary. It assumes that the curves are piecewise smooth. Each cell must be connected with unit area. There can be no interstices between the cells. Why are disks $B(0, r)$ used for the truncation? Why must the inequality involve lim sup?

We present a second version (1-B) of the theorem that has weaker hypotheses. Before stating the theorem, we discuss the form such a theorem might take. Let $T_{1}, \ldots, T_{k}, \ldots$ be a countable sequence of disjoint subsets of $\mathbb{R}^{2}$, representing the cells of a general cluster. It is natural to assume that for each $i$, the topological boundary of $T_{i}$ has finite one-dimensional Hausdorff measure. By a result of Federer, this implies that $T_{i}$ is measurable and that the current boundary $\partial T_{i}$ is rectifiable (see Section 2.1 of [M2] and Sections 4.5.12 and 2.10.6 of [F]). In general, the one-dimensional Hausdorff measure of $\bigcup_{i} \partial T_{i}$ will be infinite. To get a finite perimeter, we truncate by fixing a compact set $K \subset \mathbb{R}^{2}$ (for example, a disk of radius $\rho$ ). Let $R_{i} \subset T_{i} \cap K$ be such that $\mathcal{H}^{1}\left(\partial R_{i} \backslash \partial T_{i}\right)=0$, where $\mathcal{H}^{1}$ is the one-dimensional Hausdorff measure. For example, we could take $R_{i}$ to be the union of connected components of $T_{i}$ contained in $K$. We can measure the characteristics of the candidate $\left\{T_{i}\right\}$ through the Hausdorff measure of the sets $\bigcup \partial R_{i}$, and by taking a compact exhaustion of the plane with sets $K$. To state the honeycomb inequality, it is not necessary to refer to the original cells $T_{i}$; it can be formulated in terms of $R_{i}$ and $K$, where we now allow the area of $R_{i}$ to be less than 1 . This provides motivation for the honeycomb problem in the following general form.

Theorem 1-B (Honeycomb Conjecture for Disconnected Regions). Let $K$ be a compact set in the plane containing disjoint measurable sets $R_{1}, R_{2}, \ldots$ Assume that each $R_{i}$ has a rectifiable current boundary $\partial R_{i}$. Set $\alpha_{i}=\min \left(1\right.$, area $\left.\left(R_{i}\right)\right)$. Set $\Gamma=\bigcup_{i} \partial R_{i}$. Assume $\alpha_{i}>0$ for some $i$. Then

$$
\mathcal{H}^{1}(\Gamma)>\sqrt[4]{12} \sum \alpha_{i}
$$

Asymptotically, this inequality is sharp. For example, take $T_{i}$ to be the regions of the honeycomb tile, and $K=B(0, \rho)$, a disk of radius $\rho$. Take $R_{i}=T_{i}$, if $T_{i} \subset B(0, \rho)$, and $R_{i}=\emptyset$, otherwise. Then $\alpha_{i}=0$ or 1 , and $\sum \alpha_{i}$ is the number (or area) of the hexagonal 


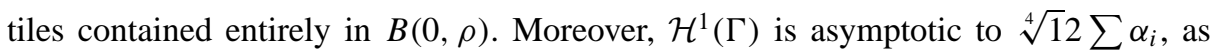
$\rho \mapsto \infty$.

We combine regions $R_{i}$ if the sum of their areas is less than 1 . This does not change $\mathcal{H}^{1}(\Gamma)$ or $\sum \alpha_{i}$, so that the validity of the theorem is not affected by combining regions. Regions can be combined as long as there are at least two regions with area between 0 and $\frac{1}{2}$. This allows us to assume without generality, when we prove Theorem 1-B, that there are only finitely many $R_{1}, \ldots, R_{n}$.

To apply regularity results, we consider an optimization problem that will lead to a lower bound on $\mathcal{H}^{1}(\Gamma)$. We fix the constants $\alpha_{i} \in[0,1]$, for $i=1, \ldots, n$. Consider the optimization problem of varying $R_{1}, \ldots, R_{n}$ so as to minimize

$$
\mathcal{H}^{1}\left(\bigcup \partial R_{i}\right)
$$

subject to the constraint that area $\left(R_{i}\right) \geq \alpha_{i}$ for $i=1, \ldots, n$. (We no longer require that $R_{i} \subset K$.)

By the existence and regularity results of [M2], there exists $\Gamma=\Gamma\left(\alpha_{1}, \ldots, \alpha_{n}\right)$ that minimizes the one-dimensional Hausdorff measure of the boundary $\bigcup \partial R_{i}$ of the corresponding regions $R_{i}$. The boundary $\Gamma$ consists of finitely many arcs of circles (possibly reducing to straight lines) meeting at vertices of degree 3. (Morgan formulates the optimization problem with equality constraints area $\left(R_{i}\right)=\beta_{i}$, where $\beta_{i} \geq \alpha_{i}$ are fixed areas. To get the existence and regularity of $\Gamma\left(\alpha_{1}, \ldots\right)$ from this, we apply Morgan's optimization to a set of constants $\beta_{i} \geq \alpha_{i}$ giving the shortest perimeter.) The minimizing set $\Gamma$ is connected [ $\mathrm{CHH}^{+}$]. Each connected component of each $R_{i}$ is simply connected. Let $R_{0}$ be the union of the connected components of $\mathbb{R}^{2} \backslash \Gamma$, other than the components of $R_{1}, \ldots, R_{n} . R_{0}$ is connected (otherwise remove edges between $R_{i}$ and $R_{0}$ to shorten $\Gamma$ and increase area $\left.\left(R_{i}\right)\right)$.

Theorem 2 (Honeycomb Conjecture, Finite Version). Assume $0<\alpha_{i} \leq 1$ for $i=$ $1, \ldots, n$. Let $A=\sum_{i} \alpha_{i}$. Then

$$
\operatorname{perim}\left(\Gamma\left(\alpha_{1}, \ldots, \alpha_{n}\right)\right)>A \sqrt[4]{12}
$$

Remark 2.1. By an argument in [M4], the case $A=\sum_{i} \alpha_{i} \leq 398$ is elementary. Assume we have $R_{1}, \ldots, R_{n}$ with $\operatorname{area}\left(R_{i}\right) \geq \alpha_{i} \in[0,1]$. Applying the isoperimetric inequality to each $R_{i}$ and then applying it once again to the union of the $R_{i}$, we find

$$
\begin{aligned}
2 \operatorname{perim}\left(\Gamma\left(\alpha_{1}, \ldots, \alpha_{n}\right)\right) & \geq \sum 2 \sqrt{\pi \alpha_{i}}+2 \sqrt{\pi A} \\
& \geq 2(\sqrt{\pi}+\sqrt{\pi} / \sqrt{A}) A \\
& >2 \sqrt[4]{12} A .
\end{aligned}
$$

Lemma 2.2 (Morgan). Theorem 2 implies Theorem 1-A.

Proof. We consider the particular case $\alpha_{1}=\cdots=\alpha_{n}=1$. Let

$$
\rho_{n}=\operatorname{perim}(\Gamma(1, \ldots, 1)) / n \text {. }
$$


Let $\rho_{\infty}$ be the infimum of the left-hand side of the inequality of Theorem 1, as $C$ runs over all planar clusters satisfying the conditions of the theorem. Let $C$ be the hexagonal honeycomb tiling to see that $\sqrt[4]{12} \geq \rho_{\infty}$. Theorem 2 gives

$$
\liminf _{n} \rho_{n} \geq \sqrt[4]{12} \geq \rho_{\infty}
$$

By Section 2.1 of [M4], we have $\rho_{\infty} \geq \liminf _{n} \rho_{n}$. The result follows.

Remark 2.3. In the third edition of [M3], Morgan extends his truncation lemma [M4, Section 2.1] to areas less than 1. This permits a generalization of Theorem 1-A to cells that are not connected.

Lemma 2.4. Theorem 2 implies Theorem 1-B.

Proof. If $\Gamma$ is the current boundary in Theorem 1-B, then the optimization problem described above yields $\Gamma\left(\alpha_{1}, \ldots, \alpha_{n}\right)$. Its perimeter gives a lower bound on the onedimensional Hausdorff measure of $\Gamma$.

Remark 2.5. Assume that $\Gamma$ is a finite connected collection of analytic arcs, and that $\Gamma$ is the boundary of (finite unions of) simply connected bounded regions $R_{i}$. Let $\alpha_{i}=$ $\min \left(1, \operatorname{area}\left(R_{i}\right)\right)$, and set $A=\sum_{i} \alpha_{i}$. Set

$$
F(\Gamma, A)=\operatorname{perim}(\Gamma)-A \sqrt[4]{12}
$$

Theorem 2 is false iff $F(\Gamma, A) \leq 0$ for some $\Gamma, A$. To check Theorem 2, we can therefore make a finite number of modifications that decrease the value of $F(\Gamma, A)$.

Remark 2.6. We claim that for the proof of Theorem 2, we can assume that the regions $R_{i}$ are connected. Each $R_{i}$ is a disjoint union of connected components $R_{i j}$. Let $\alpha_{i j}=$ $\min \left(1, \operatorname{area}\left(R_{i j}\right)\right)$ and $A^{\prime}=\sum_{i j} \alpha_{i j}$. We have $A \leq A^{\prime}$. Apply Remark 2.5.

Remark 2.7. Let $a_{0}=2 \pi \sqrt{3} / 3=4 \pi / \pi_{6}$. If $R$ is one of the regions, let $M=$ $M(R)$ be the number of sides that its boundary has (counted by the number of different regions neighboring $R$ along analytic arcs). We claim that we may assume that each $R$ satisfies

$$
\operatorname{area}(R) \geq a_{0} / M^{2}
$$

In fact, let $R$ be a region of area $a$ less than $a_{0} / M^{2}$. By the isoperimetric inequality, the perimeter of $R$ is at least $2 \sqrt{\pi a}$, so it shares at least the length $(2 / M) \sqrt{\pi a}$ with some neighboring region. Let $\Gamma^{\prime}$ be the collection of analytic arcs obtained by deleting the edges shared with this neighboring region. By deleting these edges, we "pop" the bubble $R$ and combine it with the neighboring region, so that $\Gamma^{\prime}$ bounds one fewer region than $\Gamma$. If $A^{\prime}$ is the sum of the $\alpha_{i}$ with respect to $\Gamma^{\prime}$, then $A^{\prime} \geq A-a$. We claim that 
$F(\Gamma, A)>F\left(\Gamma^{\prime}, A^{\prime}\right)$. In fact,

$$
\begin{aligned}
F(\Gamma, A)-F\left(\Gamma^{\prime}, A^{\prime}\right) & =\operatorname{perim}(\Gamma)-\operatorname{perim}\left(\Gamma^{\prime}\right)+\left(A^{\prime}-A\right) \sqrt[4]{12} \\
& \geq(2 / M) \sqrt{\pi a}-a \sqrt[4]{12} \\
& =\sqrt{a}((2 / M) \sqrt{\pi}-\sqrt{a} \sqrt[4]{12}) \\
& >\sqrt{a} M^{-1}\left(2 \sqrt{\pi}-\sqrt{a_{0}} \sqrt[4]{12}\right) \\
& =0 .
\end{aligned}
$$

Deleting edges in this way may lead to regions that are not simply connected. However, when an edge is deleted, arbitrarily short edges can be added to make the regions simply connected again. (Translate the boundary components of $\Gamma$ so that they are arbitrarily close to one another, and then connect them with a short edge.) We can choose this short edge to be so short that this modification to $\left(\Gamma^{\prime}, A^{\prime}\right)$ still gives a value of $F$ less than the original $F(\Gamma, A)$.

\section{Honeycombs on a Torus}

Let $\mathbb{R}^{2} / \Lambda$ be a torus of area at least 1 . Take a partition of the torus into a finite number of simply connected regions. Assume that the boundary consists of a finite number of simple rectifiable curves, meeting only at endpoints.

In view of Remark 2.7, we define $a(M)=\min \left(2 \pi \sqrt{3} /\left(3 M^{2}\right), 1\right)$. Assume the connected, simply connected regions are $R_{1}, \ldots, R_{n}$, and assume that $\operatorname{area}\left(R_{i}\right) \geq a\left(M_{i}\right)$.

Theorem 3 (Honeycomb Conjecture on a Torus).

$$
\operatorname{perim}\left(\bigcup \partial R_{i}\right) \geq \sum_{i=1}^{n} \alpha_{i} \sqrt[4]{1} 2
$$

Equality is attained if and only if every $R_{i}$ is a regular hexagon of area 1 and each $\alpha_{i}=1$.

This follows as an immediate consequence of an isoperimetric inequality proved in the next section. The existence of a honeycomb tiling depends on the shape and size of the lattice: $\Lambda$ must be a sublattice of a lattice formed by the tiling by unit area regular hexagonal tiles. In particular, the area of $\mathbb{R}^{2} / \Lambda$ must be an integer.

Lemma 3.1. This theorem implies the honeycomb conjecture for a finite number of cells (Theorem 2).

Proof. Consider $\left(\Gamma\left(\alpha_{1}, \ldots, \alpha_{n}\right), A=\sum \alpha_{i}\right)$ appearing in Theorem 2. By Remarks 2.6 and 2.7 , we may modify the example so that the regions in the example are connected, simply connected, and such that each region $R$ satisfies the inequality

$$
\operatorname{area}(R) \geq 2 \pi \sqrt{3} /\left(3 M^{2}\right) \geq a(M) .
$$


Let $\left(\Gamma^{\prime}, A^{\prime}\right)$ denote this modification. If we have $A^{\prime} \leq 398$, then Theorem 2 follows from Remark 2.1. Assume that $A^{\prime} \geq 398$. To complete the proof of the lemma, we move the cluster to the torus. This involves adding an additional edge of length $\sqrt{1 / A^{\prime}}$ and an additional region of area at least 1 . Theorem 3 applied to this situation gives the inequality

$$
\operatorname{perim}\left(\Gamma^{\prime}\right)+\sqrt{1 / A^{\prime}} \geq\left(1+A^{\prime}\right) \sqrt[4]{12} .
$$

When $A^{\prime} \geq 398$, this yields the inequality of Theorem 2 .

Before moving the cluster to a torus, we first move it to a cylinder. Pick a diameter to the cluster (a segment between maximally separated points $p_{1}$ and $p_{2}$ on $\Gamma$ ). Then move the cluster to the cylinder $\mathbb{R}^{2} / \mathbb{Z} v$, where $v$ is the translation along the length of the diameter. The map of the cluster to the cylinder is injective, except at the points $p_{1}$ and $p_{2}$, which become identified. Since $p_{1}$ and $p_{2}$ are maximally separated, the cluster fits inside a square of edge length $|v|$ with a pair of sides parallel to $v$. Thus the area of the cluster is at most the area $|v|^{2}$ of the square. To simplify notation, we now drop the primes from $\left(\Gamma^{\prime}, A^{\prime}\right)$. This gives $|v| \geq \sqrt{A}$, with $A=\sum \alpha_{i}$, and $\alpha_{i}=\min \left(1, \operatorname{area}\left(R_{i}\right)\right)$.

Let $w$ be a unit vector perpendicular to $v$. Pick $\mu>0$ to be the largest real number for which $\Gamma+\mu w$ touches $\Gamma$ without overlap. Let

$$
\Lambda=\mathbb{Z} v+\mathbb{Z}(\mu+\sqrt{1 / A}) w .
$$

The cluster descends to $\mathbb{R}^{2} / \Lambda$, injective except at $p_{1}$ and $p_{2}$. We add a segment of length $\sqrt{1 / A}$ to join the cluster with its translate. On the torus, the region "at infinity" becomes simply connected. Call it $R_{0}$. By adding this extra edge, we avoid the complications of a component with a loop representing a nontrivial homology class in $\mathbb{R}^{2} / \Lambda$.

Since $|v| \geq \sqrt{A}$, and the component $R_{0}$ has height at least $\sqrt{1 / A}$ in the direction of $w$ at every point, the area of $R_{0}$ is at least 1 . Now we have a partition of the torus $\mathbb{R}^{2} / \Lambda$ into connected, simply connected regions of total area at least $1+A$.

\subsection{Torus Modifications}

The combinatorial structure is described by a finite torus graph in which each face is simply connected.

Loops (edges joined at both ends to the same vertex) can be eliminated from the graph as follows. If the vertex has degree greater than 3 , then it can be considered a limit of multiple vertices of degree 3 and edges of length 0 . This can be done in such a way that the vertex on the loop has multiplicity at least 2 . If the vertex has degree 3 , let $e$ be the other edge that meets the loop at the vertex. Both sides of the edge $e$ bound the same region $E$. Removing $e$ leads to a nonsimply connected component. The loop $\Gamma_{1}$ can be moved arbitrarily close to another boundary component $\Gamma_{2}$. It can be joined to $\Gamma_{2}$ with two edges (one edge moving from a point vertex $v_{1}$ of $\Gamma_{1}$ down to $w$ on $\Gamma_{2}$, and another edge moving back to $v_{2}$ in the opposite direction, as in Fig. 1). This decreases the area of $E$, which is not permissible. However, by scaling the entire torus by a homothety $(x+\Lambda \mapsto t x+t \Lambda)$, its area is restored. Since we can make the decrease in area arbitrarily small, we can make the increase in perimeter due to the homothety arbitrarily small. In particular, we can arrange that the total increase in perimeter by this 


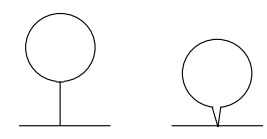

Fig. 1

process is offset by the length of the edge $e$ that was removed. (Alternatively, we could let $v_{1}=v_{2}$, and let the return path to $v_{1}$ be the same as the path from $v_{1}$ to $w$ in the opposite direction. Then $v_{1}$ is considered to be a vertex of multiplicity 2, etc.)

If the torus graph has any vertices of degree greater than 3 , we view them as vertices of degree 3 with degenerate edges of length 0 . We can do this in a way that avoids creating any new loops. Each vertex has degree 3 .

We are now in the situation where the boundary of every region $P$ is a combinatorial $N$-gon, for some $N \geq 2$, where $N=N(P)$ is the number of directed edges bounding the region. (The boundary of $P$ might traverse a segment twice in opposite directions.) We have the Euler characteristic of the torus graph

$$
0=V-E+F=\sum_{P}(1-N(P) / 6)
$$

where $P$ runs over the regions.

\section{A Hexagonal Isoperimetric Inequality for Closed Plane Curves}

Let $\Gamma$ be a closed piecewise simple rectifiable curve in the plane. In our application we take $\Gamma$ to be a lift from the torus to the plane of one of the $\partial R_{j}$ from Section 3.

We use the parametrization of the curve to give it a direction, and use the direction to assign a signed area to the bounded components of the plane determined by the curve. For example, if $\Gamma$ is a piecewise smooth curve, the signed area is given by Green's formula

$$
\int_{\Gamma} x d y
$$

Generally, we view $\Gamma$ as an integral current [M3, p. 44]. We let $P$ be an integral current with boundary $\Gamma$. (In applications, $P=R_{j}$, for some $j$.) Expressed differently, we give a signed area by assigning a multiplicity $m(U) \in \mathbb{Z}$ to each bounded component $U$ of $\mathbb{R}^{2} \backslash \Gamma$. (An illustration appears in Fig. 2.) The area is $\sum m(U)$ area $(U) . P$ is represented by the formal sum $P=\sum m(U) U$.

Let $v_{1}, \ldots, v_{t}, t \geq 2$, be a finite list of points on $\Gamma$. We do not assume that the points are distinct. Index the points $v_{1}, v_{2}, \ldots, v_{t}$, in the order provided by the parametrization of $\Gamma_{i}$. Join $v_{i}$ to $v_{i+1}$ by a directed line segment $f_{i}$ (take $v_{t+1}=v_{1}$ ). The chords $f_{i}$ form a generalized polygon, and from the direction assigned to the edges, it has a signed area $A_{P} \in \mathbb{R}$.

Let $e_{i}$ be segment of $\Gamma$ between $v_{i}$ to $v_{i+1}$. Let $f^{\text {op }}$ be the chord $f$ with the orientation reversed. Let $x\left(e_{i}\right) \in \mathbb{R}$ be the signed area of the integral current bounded by $\left(e_{i}, f_{i}^{\mathrm{op}}\right)$. Let $E(P)=\left\{e_{i}\right\}$ denote the set of edges of $P$. Accounting for multiplicities and orientations, 


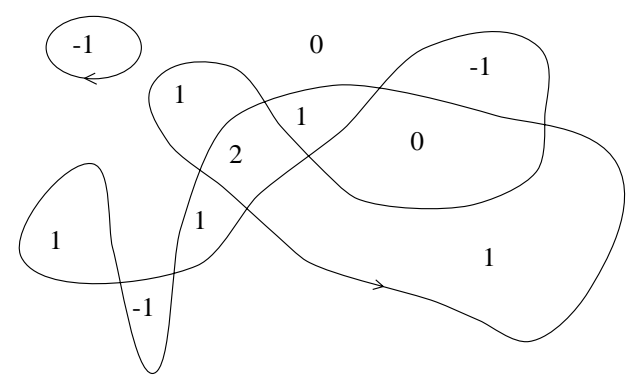

Fig. 2

we have

$$
\operatorname{area}(P)=A_{P}+\sum_{e \in E(P)} x(e) .
$$

Let $\alpha(P)=\min (1, \operatorname{area}(P))$.

Define a truncation function $\tau: \mathbb{R} \rightarrow \mathbb{R}$ by

$$
\tau(x)= \begin{cases}\frac{1}{2}, & x \geq \frac{1}{2} \\ x, & |x| \leq \frac{1}{2} \\ -\frac{1}{2}, & x \leq-\frac{1}{2}\end{cases}
$$

Set $\tau_{0}=\frac{1}{2}$. Set $T(P)=\sum_{E(P)} \tau(x(e))$. Recall that the perimeter of a regular hexagon of unit area is $2 \sqrt[4]{12}$. Let $L(P)$ be the length of $\Gamma$. Let $N(P)$ be the number of points $v_{i}$ on $\Gamma$, counted with multiplicities. Recall from Remark 2.7 that $a(N)=\min \left(2 \pi \sqrt{3} /\left(3 N^{2}\right), 1\right)$.

Theorem 4 (Hexagonal Isoperimetric Inequality). Define $P, L(P), N(P)$, and $a(N)$ as above. Assume that the signed area of $P$ is at least $a(N(P))$. Then

$$
L(P) \geq-T(P) \sqrt[4]{12}-(N(P)-6) 0.0505+2 \alpha(P) \sqrt[4]{12} .
$$

Equality is attained if and only if $P$ is a regular hexagon of area 1.

The theorem will be proved below.

Lemma 4.1. The hexagonal isoperimetric inequality implies the honeycomb conjecture for a torus (Theorem 3).

Proof. We apply this inequality to the $n$ different regions $R_{1}, \ldots, R_{n}$ in the torus partition. We let the points $v_{i}$ be the endpoints of the simple curves described in Section 3 .

Let $P$ be one of the regions $R_{i}$. The regions in Theorem 3 satisfy area $(P) \geq a(M(P))$, where $M$ is the number of different regions bounding $P$. We have $N \geq M$, so $\operatorname{area}(P) \geq$ $a(M(P)) \geq a(N(P))$. Thus, $P$ satisfies the area constraint of the hexagonal isoperimetric inequality. 
Each edge of $P$ occurs with opposite orientation $e^{\text {op }}$ on a neighboring region $P^{\prime}$, and by construction $x(e)+x\left(e^{\mathrm{op}}\right)=0$. Thus, summing over all directed edges of the partition, we get

$$
\sum_{e} x(e)=0 .
$$

Since $\tau$ is an odd function, we have $\tau(x(e))+\tau\left(x\left(e^{\mathrm{op}}\right)\right)=0$, so also

$$
\sum_{e} \tau(x(e))=\sum_{P} T(P)=0 .
$$

By Theorem 4,

$$
\begin{aligned}
2 \operatorname{perim}(\Gamma) & =\sum_{P} L(P) \\
& \geq-\sqrt[4]{12} \sum_{P} T(P)+6(0.0505) \sum_{P}(1-N(P) / 6)+2 \sum_{P} \alpha(P) \sqrt[4]{12}
\end{aligned}
$$

Thus, the lemma follows from Euler (3.1) and (4.1).

Set

$$
\begin{aligned}
\Delta(P) & =L(P)+\varepsilon(N(P), \alpha(P), T(P)) \\
\varepsilon(N, \alpha, T) & =T \sqrt[4]{1} 2+(N-6) 0.0505-2 \alpha \sqrt[4]{12} \\
X(P) & =\sum_{E(P)} x(e)
\end{aligned}
$$

Remark 4.2. The inequality is false without the truncation. For example, let $P$ be constructed as a simple closed curve of area 1 bounded by three inverted circular arcs of the same curvature and the same length (Fig. 3). When the circular arcs are sufficiently long, $L(P)+\varepsilon(3,1, X(P))<0$.

Remark 4.3. If the region $P$ is a polygon of area 1 with $v_{i}$ as vertices, then $\alpha(P)=1$, $x(e)=0$ for all $e$, and $T(P)=0$. The inequality in this case is essentially the one used by Phelan and Weaire in [P2].

Remark 4.4. The sharp case of the inequality occurs for $N(P)=6, \alpha(P)=1$, and $x(e)$ near 0 , so that $X(P)=T(P) . y=-\varepsilon(6,1, X)$ is the tangent line to $y=L\left(P_{X}\right)$ at

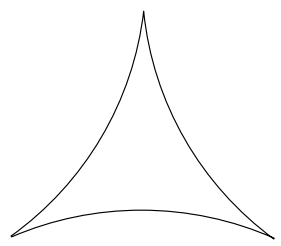

Fig. 3 

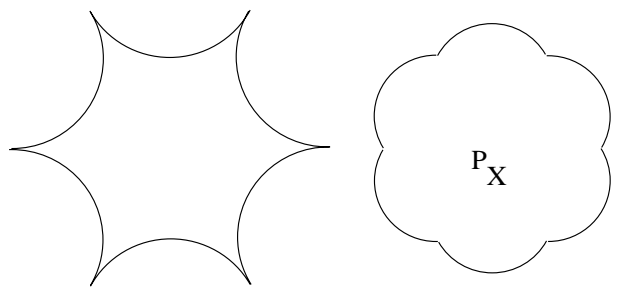

Fig. 4

$X=0$ of the following region $P_{X}$ (Figs. 4 and 5). Take a regular hexagon of area $1-X$ and add six circular arcs of the same curvature to the six edges to make the total area 1 (inverting the arcs if $X<0$ ).

\section{Preliminaries for the Proof}

Without loss of generality, we modify $P$ to decrease the perimeter, maintaining the lower bound on the area, and holding $\varepsilon$ fixed, or we may also modify $P$ by fixing the perimeter, maintaining the lower bound on the area, and decreasing $\varepsilon$.

For each chord $f$, we may apply the isoperimetric inequality to the curve (e, $\left.f^{\text {op }}\right)$ to replace $e$ with a circular arc with the same enclosed signed area. The isoperimetric inequality for integral currents appears in Section 4.5.14 of [F]. Uniqueness and regularity follow along the lines of [M2].

We may replace the polygon $\left(f_{1}, \ldots, f_{N}\right)$ by a convex polygon with the same edge lengths in the same order, that has at least the area as the original polygon. Thus, we may assume that the polygon has positive area.

If any $x(e)<-\tau_{0}$, we decrease the curvature of $e$ until $x(e)=-\tau_{0}$. This leaves $T(P)$ unchanged, decreases the perimeter, and increases area $(P)$. Thus, there is no loss in generality, if we assume $x(e) \geq-\tau_{0}$ for all $e$.

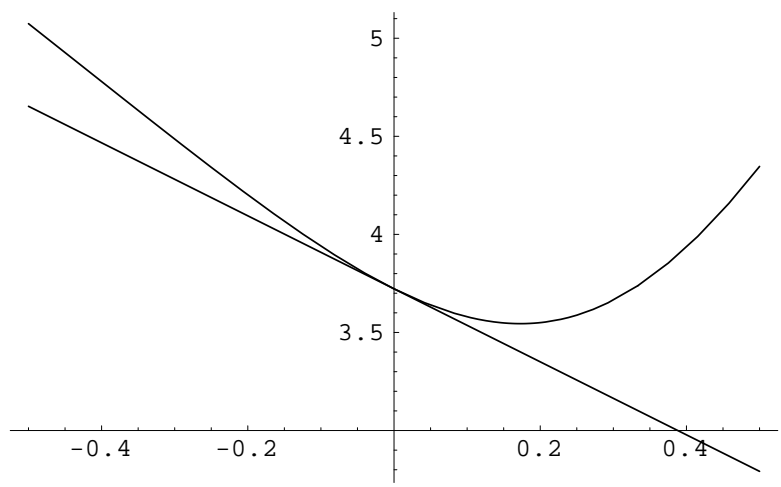

Fig. 5. The graph of $y=L\left(P_{X}\right)$ and its tangent line $y=-\varepsilon(6,1, X)$. 

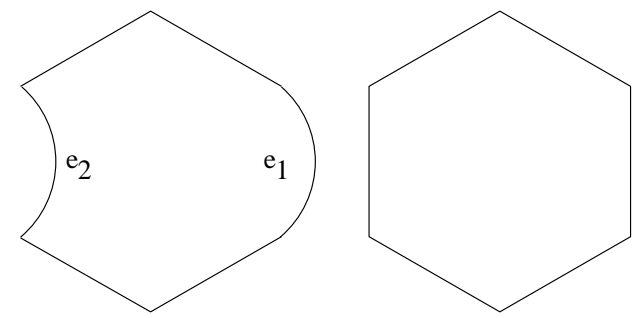

Fig. 6. A deformation reducing the perimeter at fixed area.

We leave edges $e$ with $x(e)>\tau_{0}$ untouched.

If there are two edges $e_{1}, e_{2}$ of $P$ with $0<x\left(e_{1}\right) \leq \tau_{0}$ and $x\left(e_{2}\right)<0$ (and so also $\left.x\left(e_{2}\right) \geq-\tau_{0}\right)$, we deform $P$ by decreasing the curvature of the arcs $e_{1}$ and $e_{2}$ preserving $x\left(e_{1}\right)+x\left(e_{2}\right)$, thereby decreasing the perimeter $L(P)$ (see Fig. 6). $T(P)$ is unchanged. Continuing in this way, we may assume without loss of generality that all $x(e) \in\left[-\tau_{0}, \tau_{0}\right]$ have the same sign. We consider two cases depending on whether there is an edge $e$ with $x(e)>\tau_{0}$.

Case I. For all $e, x(e) \geq-\tau_{0}$, and there exists $e$ such that $x(e)>\tau_{0}$. All $x(e) \in\left[-\tau_{0}, \tau_{0}\right]$ have the same sign.

Case II. For all $e,|x(e)| \leq \tau_{0}$. All $x(e)$ have the same sign.

The theorem will be proved by a separate argument for the two cases.

\section{Lower Bounds on $L(P)$}

We were not able to find a single estimate of $L(P)$ that leads to the theorem in all cases. Instead, we rely on number of lower bounds on $L(P)$ (and $\Delta(P)$ ). Most are based on the isoperimetric inequality.

Lower Bound $\boldsymbol{L}(\boldsymbol{N}, \alpha, \boldsymbol{X}), \boldsymbol{N}=3,4, \ldots$ The perimeter of a regular $N$-gon of area 1 is $2 \sqrt{\pi_{N}}$, where $\pi_{N}=N \tan (\pi / N)$. The polygon $\left(f_{1}, \ldots, f_{N}\right)$ has area at least $\alpha-X$. By the isoperimetric inequality for polygons, it has perimeter at least

$$
L(N, \alpha, X):=2 \sqrt{(\alpha-X) \pi_{N}} .
$$

Each $\operatorname{arc} e_{i}$ has length at least that of $f_{i}$, so $L(P) \geq L(N, \alpha, X(P))$.

Lower Bound $\boldsymbol{L}_{+}$. By the isoperimetric inequality,

$$
L(P) \geq L_{+}(\alpha):=2 \sqrt{\pi \alpha},
$$

the perimeter of a circle of area $\alpha$.

Lower Bound $\boldsymbol{L}_{-}$. Let $J$ be the set of indices of the edges with $x\left(e_{j}\right)<0$, for $j \in J$. Let $X_{J}=\sum_{J} x\left(e_{j}\right)$. By reflecting each edge $e_{j}, j \in J$, across the chord $f_{j}, P$ is replaced 
with a region of the same perimeter and area at least $\alpha-2 X_{J}$. By the isoperimetric inequality,

$$
L(P) \geq L_{-}\left(\alpha, X_{J}\right)=2 \sqrt{\left(\alpha-2 X_{J}\right) \pi} .
$$

Lower Bound $\boldsymbol{L}_{D}$. Assume here that $|x(e)| \leq \tau_{0}$, for each $e$. Dido found the curve of minimum length with both endpoints on a given line, subject to the condition that the curve and the line bound a given area. The solution is a semicircle cut through the center by the line. Applying this to an $\operatorname{arc} e$, we find that the length of $e$ is at least $\sqrt{2 \pi|x(e)|} \geq|x(e)| \sqrt{2 \pi / \tau_{0}}$. Thus,

$$
L(P) \geq L_{D}\left(X_{D}\right):=X_{D} \sqrt{2 \pi / \tau_{0}},
$$

where $X_{D}=\sum_{e}|x(e)|$. In Case II, $X_{D}=|T|=|X|$.

Lower Bound $\boldsymbol{L}^{\prime}(\boldsymbol{N}, \alpha, \boldsymbol{X})$. Only this last bound is new. For $\ell, x \geq 0$, let $\operatorname{arc}(\ell, x)$ be the length of a circular arc chosen so that together with a chord of length $\ell$ joining its endpoints, the enclosed area is $x$. For example, $\operatorname{arc}(\ell, 0)=\ell$ and $\operatorname{arc}(0, x)=2 \sqrt{x \pi}$. Let $L(N, \alpha, x)$ be as above. Let

$$
L^{\prime}(N, \alpha, X)=L(N, \alpha, X) \operatorname{arc}(1,|X| / L(N, \alpha, X))
$$

Proposition 6.1. If all the chords $f_{i}$ of $P$ have length at most 1 , if $|X| \leq 0.119$, $0.996 \leq \alpha \leq 1$, and if $N \leq 7$, then

$$
L(P) \geq L^{\prime}(N, \alpha, X),
$$

where $X=X(P), N=N(P), \alpha=\alpha(P)$.

This is proved in the Appendix.

\subsection{Equal Curvature Condition}

Here is a simple observation about the lower bounds on perimeters that we refer to as the equal curvature condition. (A version for polygons was known to Zenodorus [H, p. 210].)

Suppose that we have two chords $f_{1}$ and $f_{2}$ of circular $\operatorname{arcs} e_{1}$ and $e_{2}$. Minimize the sum of the lengths of $e_{1}$ and $e_{2}$, fixing $f_{1}$ and $f_{2}$, and constrained so the sum of the two enclosed areas is fixed. Two arcs of equal curvature give the minimum. If an arc is more than a semicircle, it occurs along the chord of greater length (or one of the two if the chords are equal in length).

To see this result, form a triangle with the two chords and a third edge of variable length $t$. Adjust $t$ until the circumscribing circle gives arcs of the correct combined enclosing area on the two chords. Any shorter perimeter contradicts the isoperimetric inequality. 


\section{Case I of the Proof of the Hexagonal Isoperimetric Inequality}

Digons. Before treating Case I, we treat the case of digons separately for both Cases I and II. Here $A_{P}=0$ and $N(P)=2$, so

$$
\operatorname{area}(P)=x\left(e_{1}\right)+x\left(e_{2}\right) \geq \alpha .
$$

Also, $\alpha \geq a(N)=a(2)>\frac{1}{4}$. In Case I, $\sum x\left(e_{i}\right)=x_{\max }+x_{\min } \geq \tau_{0}-\tau_{0} \geq 0$. In Case II, $T(P)=\sum \tau\left(x\left(e_{i}\right)\right)=\sum x\left(e_{i}\right) \geq \alpha$.

If $T(P)>0.21$, then $\Delta(P)>0$, by the bound $L_{+}: L(P) \geq 2 \sqrt{\pi \alpha} \geq 2 \alpha \sqrt{\pi}$.

Assume $T(P) \leq 0.21$. We are now in Case I, so $x_{\max }>\tau_{0}=\tau_{\max }$,

$$
x_{\min } \leq \tau_{\min } \leq 0.21-\tau_{\max }=-0.29 \text {. }
$$

By reflecting the arc $e$ corresponding to $x_{\min }$ across the corresponding chord $f$, the area becomes at least $\left(\alpha-2 x_{\min }\right)$, without changing the perimeter. We then have

$$
L(P) \geq 2 \sqrt{\pi\left(\alpha-2 x_{\min }\right)} \geq 2 \sqrt{\pi(\alpha+2(0.29))} .
$$

$\Delta(P)>0$ follows.

For the rest of the proof, we assume $N(P) \geq 3$, so that, in particular, $\varepsilon(N, \alpha, T) \geq$ $\varepsilon(3, \alpha, T)$.

Case I. We assume that for all $e, x(e) \geq-\tau_{0}$ and that for some $e, x(e)>\tau_{0}$. All $x(e)$ satisfying $|x(e)| \leq \tau_{0}$ have the same sign. In treating this case, we only need to assume that $\alpha \geq 0$, rather than $\alpha \geq a(N)$.

Assume $T(P)>0.177$. We have the bounds $L_{+}: L(P) \geq 2 \alpha \sqrt{\pi}$ and $\varepsilon(N, \alpha, T) \geq$ $\varepsilon(3, \alpha, 0.177)$. It follows that $\Delta(P)>0$.

Assume next that $T(P)<-0.36$. There exists $x(e)<0$. Index so that $x\left(e_{i}\right)>\tau_{0}$ for $i \in I$ and $x\left(e_{j}\right) \leq 0$ for $j \in J$. Set $X_{I}=\sum x\left(e_{i}\right), X_{J}=\sum x\left(e_{j}\right)$, so that $X(P)=X_{I}+X_{J}$. The area of $P$ is $X_{I}+X_{J}+A_{P} \geq \alpha$. Let $k=|I|$. We have $T(P)=k \tau_{0}+X_{J}$. By Dido,

$$
L(P) \geq \sum_{I} \sqrt{2 \pi\left|x_{i}\right|}+\sum_{J} \sqrt{2 \pi\left|x_{j}\right|} \geq k \sqrt{2 \pi \tau_{0}}-X_{J} \sqrt{2 \pi / \tau_{0}} .
$$

Then

$$
\Delta(P) \geq k \sqrt{2 \pi \tau_{0}}-X_{J} \sqrt{2 \pi / \tau_{0}}+\varepsilon\left(3,1, k \tau_{0}+X_{J}\right) .
$$

Substituting the upper bound $X_{J} \leq-0.36-k \tau_{0}$ for $X_{J}$, and then the lower bound $k \geq 1$ for $k$, we find that $\Delta(P)>0$.

Assume finally that $T(P) \in[-0.36,0.177]$. With the same notation, we have $T(P)=$ $k \tau_{0}+X_{J} \leq 0.177$, which gives $X_{J} \leq-0.323$. Reflecting the arcs corresponding to negative signed areas as above, we get

$$
L(P) \geq 2 \sqrt{\pi(\alpha+2(0.323))} .
$$

This gives $\Delta(P)>0$. 


\section{Case II of the Proof of the Hexagonal Isoperimetric Inequality}

Assume that $|x(e)| \leq \tau_{0}$ for all $e$, and that all $x(e)$ have the same sign. Then $X(P)=$ $T(P)$ and $X_{D}=|X(P)|$.

First, we treat the case $\alpha \in\left[2 \pi \sqrt{3} /\left(3 N^{2}\right), \frac{1}{4}\right]$, and then we treat the case $\alpha \geq \frac{1}{4}$. If $\frac{1}{4} \geq \alpha \geq 2 \pi \sqrt{3} /\left(3 N^{2}\right)$, we have $N \geq 4$. If $T \geq 0$, we use $L(P) \geq L_{+}(\alpha)$ to get

$$
\Delta(P) \geq 2 \sqrt{\pi \alpha}+(N-6) 0.0505-2 \alpha \sqrt{\pi} .
$$

The second derivative in $\alpha$ is negative, so it is enough to check that this is positive for $\alpha=2 \pi \sqrt{3} /\left(3 N^{2}\right)$, and $\alpha=\frac{1}{4}$. This is elementary.

Next, if $T \in[-2.4,0]$, we use $L(P) \geq L_{-}(P)$. We show that the following is positive:

$$
2 \sqrt{\pi(\alpha-2 T)}+T \sqrt[4]{12}+(N-6) 0.0505-2 \alpha \sqrt[4]{12}
$$

The second derivative in $\alpha$ is negative as well as that for $T$. Hence, it is enough to check positivity for $T=0,-2.4$, and $\alpha=2 \pi \sqrt{3} /\left(3 N^{2}\right), \frac{1}{4}$. Again, the verification is elementary.

Finally, if $T \leq-2.4$, we use $L(P) \geq L_{D}$. It is clear that

$$
\Delta(P) \geq-T \sqrt{2 \pi / \tau_{0}}+\varepsilon(N, 1, T) \geq 0 .
$$

This completes our discussion of the case $\alpha \in\left[2 \pi \sqrt{3} /\left(3 N^{2}\right), \frac{1}{4}\right]$.

The rest of Section 8 is devoted to the case $\alpha \geq \frac{1}{4}$. We pick a lower bound $\tilde{L}(N, \alpha, X)$ for $L(P)$ from the stock of lower bounds developed in Section 6, according to the schematic in the $(N, X)$ plane shown in Fig. 7.

The boundary between $L_{+}(1)$ and $L(N, \cdot)$ is the curve $X_{N}^{+}=1-\pi / \pi_{N}>0$, determined by the condition $L_{+}(1)=L\left(N, 1, X_{N}^{+}\right)$. (For $N=3$, we set $X_{3}^{+}=0.177$, instead of $1-\pi / \pi_{3}$.) Also, set $X_{N}^{-}=\left(-\pi+\pi_{N}\right) /\left(-2 \pi+\pi_{N}\right)<0$, which satisfies $L_{-}\left(1, X_{N}^{-}\right)=L\left(N, 1, X_{N}^{-}\right)$. When $X<0$, we have $X=X_{J}$, and $L_{-}\left(\alpha, X_{J}\right)=$

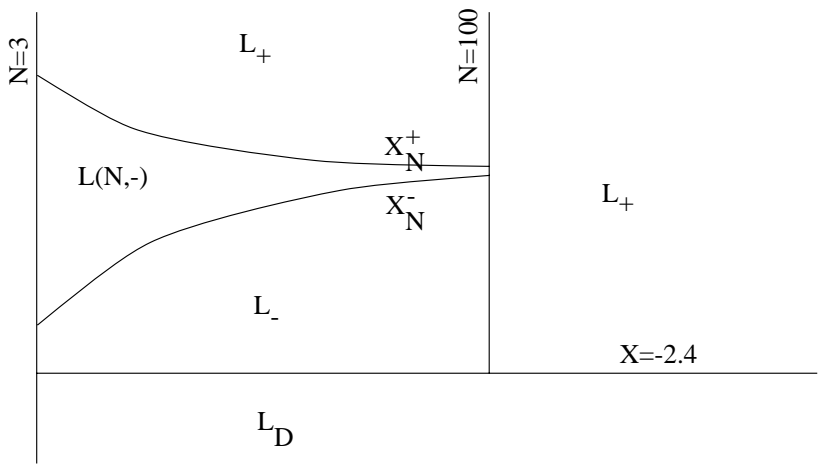

Fig. 7 


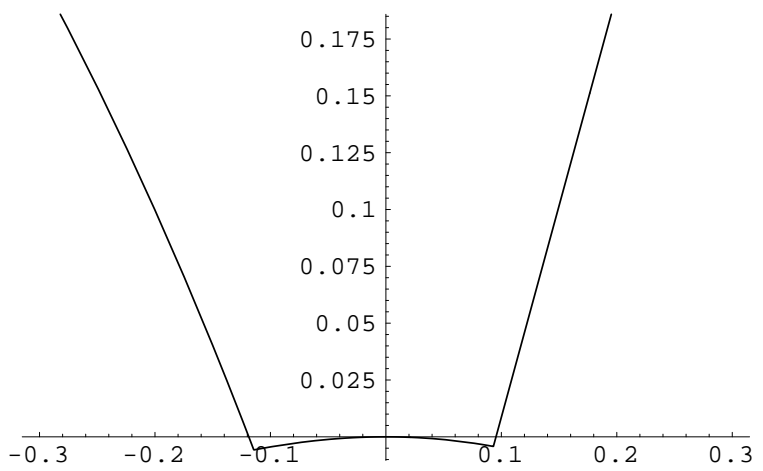

Fig. 8. The graph of $\tilde{L}(6,1, X)+\varepsilon(6,1, X)$.

$L_{-}(\alpha, X)$. We omit the details of a routine calculation that shows

$$
\tilde{L}(N, \alpha, X)+\varepsilon(N, \alpha, X) \geq \tilde{L}(N, 1, X)+\varepsilon(N, 1, X),
$$

for $N>4$.

The function $\tilde{L}(N, 1, X)+\varepsilon(N, 1, X)$ is piecewise analytic and has a negative second derivative in $X$. This makes it trivial to check that this function is positive on a given analytic interval by checking the values at the endpoints. The function $\tilde{L}(N, \alpha, X)+$ $\varepsilon(N, \alpha, X)$, for $N=3,4$, is also easily checked to be positive. We find that

$$
\Delta(P) \geq \tilde{L}(N, \alpha, X)+\varepsilon(N, \alpha, X)>0,
$$

except in the following two situations that will be treated below:

(1) $N=6, \alpha \in[0.996,1]$, and $X \in[-0.119,0.1]$,

(2) $N=7, \alpha \in[0.996,1]$, and $X \in[-0.082,0.0684]$.

Figures 8 and 9 show $\tilde{L}(N, 1, X)+\varepsilon(N, 1, X)$, for $N=6,7$.

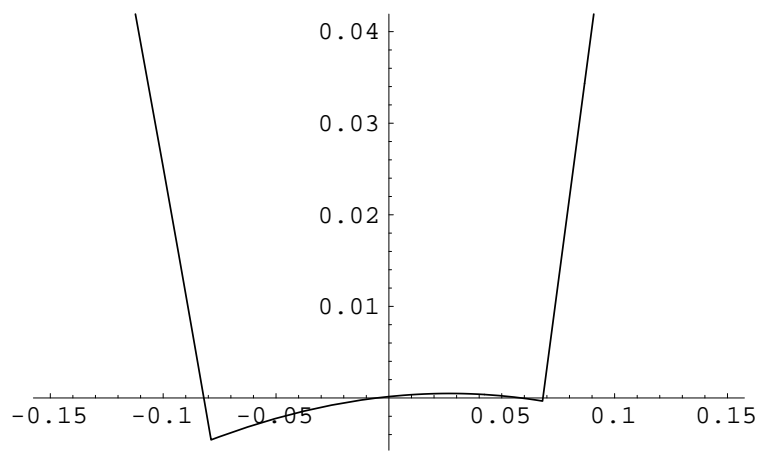

Fig. 9. The graph of $\tilde{L}(7,1, X)+\varepsilon(7,1, X)$. 
For the rest of the argument, we assume we are in one of these two situations. If the lengths of the chords $f_{i}$ are at most 1 , we have

$$
\Delta(P) \geq L^{\prime}(N, \alpha, X)+\varepsilon(N, \alpha, X) \geq 0 .
$$

The inequality on the left is Proposition 6.1.

The inequality on the right was checked by taking a Taylor approximation at $X=0$ (and by taking the worst-case values for $\alpha$ ). We give a few details of the approximation in the special case $N=6,|X| \leq 0.06$. The constant $\alpha$ satisfies $0<\alpha \leq 1$. We also assume that $0 \leq \theta \leq 0.5$, where $\theta$ is a variable defined below. The function takes the form

$$
L^{\prime}(6, \alpha, X)+\varepsilon(6, \alpha, X)=\sqrt[4]{1} 2(2 q(\theta) \sqrt{\alpha-X}-2 \alpha+X),
$$

where $q(\theta)=\theta / \sin \theta$, and $\theta \geq 0$ is defined implicitly by the equation

$$
p(\theta):=\frac{\theta-\sin \theta \cos \theta}{\sin ^{2} \theta}=\frac{2|X|}{\sqrt{\alpha-X} \sqrt[4]{12}} .
$$

(This formula for $L^{\prime}$ is presented in greater detail in the Appendix.) We show that

$$
2 q(\theta) \sqrt{\alpha-X}-2 \alpha+X \geq 0
$$

with equality exactly when $X=0$. The following inequalities are easily verified, when the variables lie in the indicated intervals:

$$
\begin{aligned}
& \sqrt{1-X} \geq 1-\frac{X}{2}-0.22 X^{2} \\
& \frac{2|X|}{\sqrt[4]{12} \sqrt{1+0.06}} \leq \frac{2|X|}{\sqrt[4]{1} 2 \sqrt{\alpha-X}}=p(\theta) \leq 0.87 \theta \text {; } \\
& q(\theta) \geq 1+\frac{\theta^{2}}{6} \geq 1+\frac{4 X^{2}}{6\left(0.87^{2}\right)(1.06) \sqrt{12}} ; \\
& 2 q(\theta) \sqrt{\alpha-X}-2 \alpha+X \\
& \geq 2\left(1+\frac{4 X^{2}}{6\left(0.87^{2}\right)(1.06) \sqrt{12}}\right)\left(1-\frac{X}{2}-0.22 X^{2}\right)-2+X=X^{2} f,
\end{aligned}
$$

where $f$ is a quadratic polynomial in $X$ taking positive values for $|X| \leq 0.06$. It follows that if the function vanishes, then $X=0$. This implies $\theta=0$, and $q(\theta)=1$. We then have

$$
L^{\prime}(6, \alpha, 0)+\varepsilon(6, \alpha, 0)=\sqrt[4]{1} 2(2 \sqrt{\alpha}-2 \alpha) .
$$

If this vanishes, then $\alpha=1$.

This completes the hardest case. Similar calculations are left to the reader when $N=7,|X| \geq 0.06$, or $\theta \geq 0.5$. The result is that $\Delta(P)=0$, if and only if $X=0$ and $P$ is a regular hexagon with area 1 . This is the tight case of the hexagonal isoperimetric 
inequality. (The graph of $y=L^{\prime}(6,1, X)$ lies between the graphs of $y=L\left(P_{X}\right)$ and $y=-\varepsilon(6,1, X)$ in Fig. 5.)

Now assume that some chord $f_{i}$ has length at least 1 . The area of the polygon $P_{f}=$ $\left(f_{1}, \ldots, f_{N}\right)$ is at least $\alpha-X$. A lower bound on $L(P)$ is the perimeter $L\left(P_{f}\right)$ of this polygon.

Lemma 8.1. $L\left(P_{f}\right)+\varepsilon(N, \alpha, X)>0$, in situations (1) and (2).

Proof. We develop an isoperimetric inequality for polygons of area at least $\alpha-X$, constrained so that one of the edges has length at least 1 . We may assume that the area is $0.996-X$. By well-known principles, the optimal polygon is inscribed in a circle with unconstrained edge lengths $t$ and constrained edge length $\max (1, t)$. The area $A_{N}(r)$ and perimeter $\max (1, t)+(N-1) t$ are monotonic increasing functions of the circle's radius $r$.

Now $X=X_{N}(r)=0.996-A_{N}(r)$, so

$$
\Delta(P) \geq g_{N}(r):=\max (1, t)+(N-1) t+\varepsilon\left(N, 1, X_{N}(r)\right) .
$$

The function $g_{N}(r)$ is easily estimated because of the monotonicity of $t$ and $A_{N}$. For $a<b$, we write

$$
g_{N}(a, b)=\max (1, t(a))+(N-1) t(a)+\varepsilon\left(N, 1,0.996-A_{N}(b)\right) .
$$

Then $\Delta(P) \geq g_{N}(r) \geq g_{N}(a, b)$, for $r \in[a, b]$. In situation (1),

$$
X_{6}(0.671)<-0.119 \leq X \leq 0.1<X_{6}(0.61) .
$$

Thus, it can be seen that $\Delta(P)>0$, by computing the constants

$$
g_{6}(0.61+0.001 k, 0.611+0.001 k)>0 \text {, }
$$

for $i=0, \ldots, 60$. (The constants are all at least 0.02.) Situation (2) is similar.

\section{Appendix. $\quad$ A Proof of Proposition 6.1}

Let $P$ have chords $f_{i}$ of length $\ell_{i}$. In this appendix we give a proof of the following result.

Proposition 6.1-A. Choose constants $s$ and $\ell$ so that $\ell_{i} \leq s<\ell \leq \sum \ell_{i}$, for all $i$. If $|X(P)| \leq \pi s^{2} / 8$, then

$$
L(P) \geq \ell \operatorname{arc}(s,|X(P)| / \ell)
$$

We obtain the version of the proposition that is stated in Section 6 by taking $s=1$, $\ell=L(N, \alpha, X(P)), N(P) \leq 7$, and $\alpha \in[0.996,1]$. Note that under these conditions, $0.119<\pi s^{2} / 8$ and $\ell \operatorname{arc}(s,|X(P)| / \ell)=L^{\prime}(N, \alpha, X)$. 


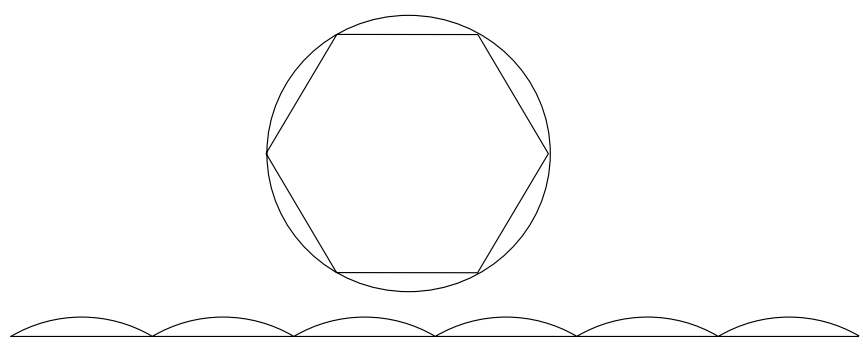

Fig. 10

Proof. We flatten out the perimeter of the polygon by arranging its edges $f_{i}$ along a line as shown in Fig. 10. Without loss of generality, we may assume that all $x(e)$ have the same sign. (See Section 5, noting that the truncation $\tau_{0}$ is not used in this appendix.)

Next we analyze two particular edges of lengths $(u, v)=\left(\ell_{i}, \ell_{j}\right)$. Let the lengths of the two circular arcs be $2 \rho \theta_{u}$ and $2 \rho \theta_{v}$, where $1 / \rho$ is common curvature of the two arcs (see Section 6.1). That is, $\theta_{u}$ is the angle subtended by the arc and its chord (Fig. 11).

Set

$$
\begin{aligned}
& p(t)=\frac{t-\sin (t) \cos (t)}{\sin ^{2}(t)}, \\
& q(t)=\frac{t}{\sin (t)}
\end{aligned}
$$

We have

$$
\begin{aligned}
\ell_{i}+\ell_{j} & =u+v, \\
4\left(\left|x\left(e_{i}\right)\right|+\left|x\left(e_{j}\right)\right|\right) & =u^{2} p\left(\theta_{u}\right)+v^{2} p\left(\theta_{v}\right), \\
\operatorname{perim}\left(e_{i}\right)+\operatorname{perim}\left(e_{j}\right) & =u q\left(\theta_{u}\right)+v q\left(\theta_{v}\right), \\
0 & =u \sin \theta_{v}-v \sin \theta_{u} .
\end{aligned}
$$

These four equations give the length of the chords, the enclosed area, the arc length of the circular arcs, and the equal curvature condition for the two edges. Let $\xi(u)$ be the area $\left|x\left(e_{i}\right)\right|+\left|x\left(e_{j}\right)\right|$, viewed as a function of $u$ by fixing the combined length $\ell_{i}+\ell_{j}$, perimeter $\operatorname{perim}\left(e_{i}\right)+\operatorname{perim}\left(e_{j}\right)$, and the equal curvature condition. It is defined implicitly by (A.1)-(A.4).

\section{Lemma A.1.}

$$
\xi^{\prime}(u)=\rho\left(\cos \theta_{v}-\cos \theta_{u}\right) .
$$

In particular, $\xi^{\prime}(u) \geq 0$, if $0 \leq \theta_{v} \leq \theta_{u} \leq \pi$.

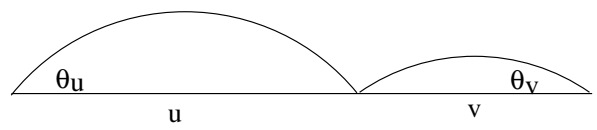

Fig. 11 
Proof. We compute the derivative $\xi^{\prime}(u)$ by implicit differentiation of (A.1)-(A.4). The differentials of these four equations give four homogeneous linear relations among $d u$, $d v, d \theta_{u}, d \theta_{v}$, and $d \xi$. Solving the linear system for $d \xi / d u$, we obtain the result.

Remark A.2. Johnson and Morgan have observed that the inequality $\xi^{\prime}(u) \geq \rho \cos \theta_{v}-$ $\rho \cos \theta_{u}$ can be seen geometrically without a calculation. This inequality is all that is needed for the proof of Proposition 6.1-A. (In fact, $\xi^{\prime} \geq 0$ is all that is needed.) The heights of the two bumps in Fig. 11 are $h\left(\theta_{u}\right), h\left(\theta_{v}\right)$, where $h(\theta)=\rho-\rho \cos \theta$. We can increase the area by

$$
\left(h\left(\theta_{u}\right)-h\left(\theta_{v}\right)\right) \Delta u,
$$

by cutting a vertical slice of area $h\left(\theta_{v}\right) \Delta u$ from the middle of the small bump, and adding a vertical slice of area $h\left(\theta_{u}\right) \Delta u$ to the middle of the large bump. To first order, this keeps the length of the perimeter constant. The optimal increase in area $\Delta \xi$ is at least the increase in area obtained by this strategy. Hence the inequality.

The lemma leads to a proof of the proposition. By the equal curvature condition (Section 6.1), if $u \geq v$, then $\theta_{u} \geq \theta_{v}$, so lengthening longer chords decreases the perimeter for fixed areas.

By continuity, it is enough to prove the proposition when $\ell=a / b$ is a rational number. We apply the lemma to pairs of chords, increasing the longer chord $u<s$ and decreasing the shorter chord $v>0$, keeping the sum $u+v$ fixed, continuing until every segment has length 0 or $s$, except for one of length between 0 and $s$.

$\pi s^{2} / 8$ is the area of a semicircle with diameter $s$. By the equal curvature condition (Section 6.1), any circular arc greater than a semicircle must lie along a chord of length $s$. The area under such an arc is greater than $\pi s^{2} / 8$, contrary to hypothesis.

If $\sum \ell_{i}>\ell$, pick an edge of length $s$. The arc $e_{i}$ along that edge is less than a semicircle. Decreasing the diameter $\ell_{i}$ while fixing $x\left(e_{i}\right)$ will decrease the length of $e_{i}$. (This is a standard argument: decrease the obtuse angle of the triangle joining the midpoint of $e_{i}$ to the endpoints of $f_{i}$. This increases the area of the triangle, keeping the perimeter fixed.) Continuing in this manner, we can decrease $\sum \ell_{i}$ until $\ell=\sum \ell_{i}$. Again, we may assume that all lengths but one are $s$ or 0 .

We replicate $b$ times these arcs and chords, enclosing a total area of at most $b \pi s^{2} / 8$. We continue to apply the lemma to pairs of chords until all edges have length 0 or $s$. At no stage do the circular arcs along the chords of length $s$ become semicircles. The perimeter of the replicated version is $a \operatorname{arc}(s, b|X| / a)$, so the perimeter of the unreplicated version is $\ell \operatorname{arc}(s,|X(P)| / \ell)$ as desired.

\section{Acknowledgments}

I thank J. Sullivan for many helpful comments. I am particularly grateful to F. Morgan for many comments, suggestions, and corrections. I thank D. Weaire for his recommendation, "Given its celebrated history, it seems worth a try ...." 


\section{References}

[A] F. J. Almgren, Jr, Existence and regularity of almost everywhere of solutions to elliptic variational problems with constraints, Mem. Amer. Math. Soc., 165 (1976).

[B] T. Bonnesen, Les problèmes des isopérimètres, 1929.

[BBC] A. Bezdek, K. Bezdek, and R. Connelly, Finite and uniform stability of sphere packings, Discrete Comput. Geom. 20 (1998), 111-130.

[CFG] H. Croft, K. Falconer, and R. Guy, Unsolved Problems in Geometry, Springer-Verlag, New York, 1991.

$\left[\mathrm{CHH}^{+}\right]$C. Cox, L. Harrison, M. Hutchings, S. Kim, J. Light, A. Mauer, and M. Tilton, The shortest enclosure of three connected areas in $\mathbb{R}^{2}$, Real Anal. Exchange 20(1) (1994/95), 313-335.

[CS] J. H. Conway and N. J. A. Sloane, What are all the best sphere packings in low dimensions? Discrete Comput. Geom. 13 (1995), 383-403.

[D] C. Darwin, On the Origin of the Species.

[F] H. Federer, Geometric Measure Theory, Springer-Verlag, New York, 1969.

[FT1] L. Fejes Tóth, Über das kürzeste Kurvennetz das eine Kugeloberfläche in flächengleiche konvexe Teil zerlegt, Mat. Term.-tud. Értesitö 62 (1943), 349-354.

[FT2] L. Fejes Tóth, Regular Figures, MacMillan, New York, 1964.

[FT3] L. Fejes Tóth, What the bees know and what they do not know, Bull. Amer. Math. Soc. 70 (1964).

[H] T. Heath, A History of Greek Mathematics, Vol II, Oxford University Press, Oxford, 1921.

[K1] J. Kepler,L'étrenne ou la neige sexangulaire, Introduction by R. Halleux, C.N.R.S., 1975.

[K2] G. Kuperberg, Notions of denseness, Preprint, math.MG/9908003.

[L1] M. Lhuilier, Mémoire sur le minimum de cire des alvéoles des abeilles, Nouveaux Mémoires de l'Académie Royale des Sciences de Berlin, 1781.

[L2] M. Lhuilier, Abrégé d'isopérimétrie élémentaire, 1789.

[M1] C. MacLaurin, Of the bases of the cells wherein the bees deposit their honey, Philos. Trans. Roy. Soc. London, 1743.

[M2] F. Morgan, Soap bubbles in $\mathbb{R}^{2}$ and in surfaces, Pacific J. Math. 165(2) (1994), 347-361.

[M3] F. Morgan, Geometric Measure Theory, A Beginner's Guide, second edition, Academic Press, New York, 1995.

[M4] F. Morgan, The hexagonal honeycomb conjecture, Trans. Amer. Math. Soc. 351(5) (1999), 17531763.

[P1] Pappus d'Alexandrie, La collection mathématique, tr. Paul Ver Eecke, Albert Blanchard, 1982.

[P2] R. Phelan, Generalisations of the Kelvin problem and other minimal problems, in [W2], 1996.

[T1] J. Taylor, The structure of singularities in soap-bubble-like and soap-film-like minimal surfaces, Ann. of Math. 103 (1976), 489-539.

[T2] D. Thompson, On Growth and Form, Cambridge University Press, Cambridge, 1952.

[V] M. T. Varro, On Agriculture, Loeb Classical Library, 1934.

[W1] H. Weyl, Symmetry, Princeton University Press, Princeton, NJ, 1952.

[W2] D. Weaire, The Kelvin Problem: Foam Structures of Minimal Surface Area, 1996.

[W3] V. Willem, L'architecture des abeilles, 1928.

Received August 30, 1999, and in revised form April 17, 2000. Online publication September 22, 2000. 